\title{
A multi-population survey on swine feeding behavior with electronic feeding devices
}

\author{
Wenshui Xin ${ }^{1, *}$, Xinjian $\mathrm{Li}^{2}{ }^{2, *}$, Feng Zhang ${ }^{1}$, Guorong Yan $^{1}$, Nengshui Ding ${ }^{1}$, Lusheng Huang ${ }^{1}$, and \\ Zhiyan Zhang ${ }^{1}$ \\ ${ }^{1}$ State Key Laboratory for Swine Genetics, Breeding and Production Technology, \\ Jiangxi Agricultural University, Nanchang, 330045, China \\ ${ }^{2}$ College of Animal Science and Veterinary Medicine, Henan Agricultural University, \\ Zhengzhou, 450002, China \\ *These authors contributed equally to this work. \\ Correspondence to: Zhiyan Zhang (bioducklily@ hotmail.com) \\ and Lusheng Huang (lushenghuang @ hotmail.com)
}

Received: 15 June 2016 - Revised: 6 September 2016 - Accepted: 17 October 2016 - Published: 1 November 2016

\begin{abstract}
In this study, we used electronic feeding station observations of pigs to investigate their growth performance and feeding behavior. These pigs were raised in Xinda Livestock Company Ltd. in Henan, China, and followed the Chinese directive for the protection of farm animals. Electronic feeding stations are often used in pig breeding and for identifying loci associated with feed consumption. Moreover, they can also provide much valuable information on pig behavior that could be directly used to improve production efficiency. This study involves three different pig population feeding-intake data from 383 Yorkshire, 243 Landrace and 197 Duroc breeds to investigate their growth performance and feeding behavior. Similar patterns of growth performance (slow-fast-slow) were observed in the three breeds, while the Duroc have a slightly faster average growth rate throughout the whole test period. Study of the number of visits to the feeding station over $24 \mathrm{~h}$ detected two peaks of feeding intake activity at 08:00-09:00 and 14:00-15:00. During these two peak feeding times, lower feed intake and less occupation time were observed, implicating fiercer competition at these time periods. The effective intake time for Duroc, Landrace and Yorkshire populations was 19, 16 and 19 min, respectively, suggesting that the ultimate feed intake time for each individual can be set up to $20 \mathrm{~min}$ for each visit. Studies on the ADFI (average daily feed intake) showed a significant difference $(P$ value $=0.000009)$ between seasons and consistent patterns for these three breeds. The present study provides a detailed survey on pig feed intake behaviors across different populations and feeding seasons.
\end{abstract}

\section{Introduction}

Since the late 1980s, electronic feeding stations have been installed to measure the feed intake of individual pigs housed in groups. This system automatically records the weight of the visitor and food intake as well as the time of entry into and exit out of the station by each pig. These raw data were finally summarized as the food conversion rate, which was used in genetic selection programs for performance testing in pigs and in identifying genetic loci responsible for feed consumption. However, the raw data recorded by the equip- ment included information on longitudinal feeding behavior of each individual and feeding patterns in group-housed animals, which could be further used for managing livestock, identifying sick animals and determining genetic differences within a herd.

Investigations on feeding behavior using electronic feeding data have been widely performed on cattle (Basarab et al., 2003; Chapinal et al., 2007; Kelly et al., 2010), swine, goat, small livestock (Basarab et al., 2003; Gipson et al., 2007; Goetsch et al., 2010) and poultry (Puma et al., 2001). Each study included combinations of parameters of feed intake, 
feeding time, feeding interval, number of feeds per day, total time spent on eating and feeding speed. The researchers found that feed intake and feeding behavior was impacted by environmental temperature (Eigenberg et al., 2000), diet (Fuller et al., 1995; Abijaoudé et al., 2000), social communication (Goetsch et al., 2010), dominance ranking (Chapinal et al., 2008; Val-Laillet et al., 2008; Walker et al., 2008; Sołtysiak and Nogalski, 2010), number of individuals in a feeder (Korthals, 2000) and health status (Griffin, 2001). However, most of these data were recorded from small groups or individually housed animals. It is better to dynamically uncover pig feeding behavior within a commercial setting. With better understanding of animal behavior and continual improvement of equipment, systems could possibly be modified to electronically monitor animals to ensure their proper care, improve their well-being, ensure the correct use of antibiotics as well as the profitability of operations.

We collected data from an electronic automatic feeder used for most popular commercial breeds with the aims to (1) detect the relationship between body weight and age in three commercial breeds, (2) investigate the feeding behavior among these breeds, (3) determine the main pattern of feeding activity to certain times of day and (4) evaluate the impact of seasons on feed intake. The results of this study will help to group numbers of pigs in a pen, take advantage of seasons for feeding pigs and utilize feeding behavior parameters to manage animals in a commercial setting.

\section{Materials and methods}

Animal care and tissue collection procedures followed the guidelines established by the Ministry of Agriculture of China. The ethics committee of Jiangxi Agricultural University specifically approved this study.

\subsection{Animals and housing conditions}

This study takes advantage of data on growth performance from a progeny test station at the Henan Xinda Livestock Company Ltd., China. The progeny test station comprises of two stables, each stable composes 24 pens, and each pen has a total area of $21 \mathrm{~m}^{2}(3 \times 7 \mathrm{~m})$ with fully slatted floor. Each pen has its own electronic feeding station and a maximum housing capacity of 12 pigs, leading to a total capacity of 576 pigs for any one time and with an average of $1.75 \mathrm{~m}^{2}$ space allowance per pig. The microclimate of the test station was controlled by a negative pressure ventilation system realizing a temperature between 16 and $22^{\circ} \mathrm{C}$ and a humidity between 60 and $70 \%$. An electronic automatic feeder (FIRE; Osborne Industries, Inc.; United States of America) was set up in the corner of the pen. When a pig entered the feeder it was automatically identified through an electronic ear tag and other pigs were prevented from entering into the feeder by a protective crate or from interacting with the pig during its feeding time. For each instance, the identification number, weight of visitor, weight of food intake, time of entry and time of exit from the feeder were recorded automatically.

Three batches of a total of 823 purebred male pigs from three commercial American breeds (Landrace, Duroc and Yorkshire) were used in this study. The dates and total number of pigs that entered the test station in each batch are 17 September-28 December 2014, 17 December 201411 April 2015 and 3 February 2015-27 May 2015 with 243, 197 and 383 pigs, respectively. They were delivered to the test station after weaning, with weights ranging from 25 to $35 \mathrm{~kg}$. Each batch includes three breeds and were randomly grouped into the pen with the number varying from 10 to 12 (mainly 12) depending on the number of available animals. Pigs had permanent access to ad libitum fresh water (nipple water) and food containing $15.5 \%$ crude protein, $3250 \mathrm{~kJ}$ of daily energy and $0.85 \%$ lysine. All diets were fortified with vitamins and minerals with respect to the age of the pig. Data from the routine herd recordings regarding breed, date of birth, test date and body weight at arrival are accessible from the electronic test machine.

\subsection{Methods and models}

With the data records - time each individual pig visited the feeder, its weight, its total feed consumption during the visit and identification number together with its birth date, test date and body weight at arrival - we extracted several indexes for further investigation using house-developed $\mathrm{R}$ scripts including (i) number of visits per day (NV), (ii) daily feed intake (DFI), (iii) occupation time per visit (OTPV) and (iv) feed intake weight per visit (FIWPV).

The analysis was performed using data once they were satisfied by several QC (quality control) thresholds. The analysis included (i) records of the FIRE after 7 days of satiation, (ii) FIWPV values larger than $10 \mathrm{~g}$ and less than $99 \%$ quantile of intake weight per visit, (iii) the residual feed weight in the feeder when less than $500 \mathrm{~g}$, and (iv) OTPV values larger than $0 \mathrm{~min}$ and less than $30 \mathrm{~min}$. The QC thresholds were empirical values that we got from the front-line managers.

With these data, the feed intake behavior included the growth performance and distributions of NV, DFI and OTPV of each of the three breeds studied. The differences among breeds and seasons of feed intake behavior were also investigated. Within the effective feed intake time calculation, we hypothesize that the feed intake weight has a high correlation with feed intake time at the start and then it gradually changes when close to zero. The effective feed intake time was defined by the beginning of feeding until the time that feed intake weight no longer increased. A general linear model was used to calculate the effective feed intake time. These calculations were also carried out using a self-coded $\mathrm{R}$ script performed using the program $\mathrm{R}$ version 3.2.5 on a Windows system. 


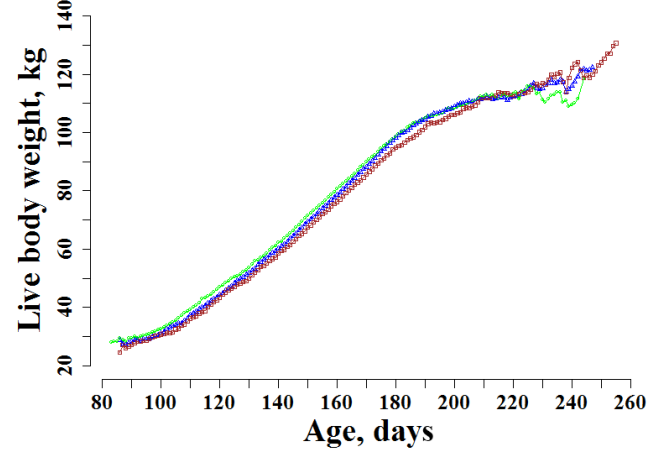

Figure 1. Relationship between live body weight, $x$ axis, and age of pig in days, $y$ axis. The brown, green and blue lines represent Duroc, Landrace and Yorkshire breeds, respectively.

Table 1. Logistic function for the three breeds studied.

\begin{tabular}{ll}
\hline Breed & Logistic function \\
\hline Duroc & $y=\frac{138}{1+34.32 e^{-0.0233 x}}$ \\
Landrace & $y=\frac{124}{1+38.17 e^{-0.0268 x}}$ \\
Yorkshire & $y=\frac{128}{1+44.40 e^{-0.0268 x}}$ \\
\hline
\end{tabular}

$y$ is body weight; $x$ is days

The logistic model was used in growth performance:

$y=\frac{K}{1+a e^{-b x}}$.

Here, $y$ is the body weight of pigs; $x$ is the age (in days); the parameters $K, a$ and $b$ were calculated using Microsoft Excel 2016, and the knee points of body weight and age (in days) were defined as $K / 2$ and $\frac{\ln a}{b}$ (Xihong et al., 2011; Yongqiang et al., 2011). Variance analysis and Tukey multiple comparisons of means were used in investigating the feed intake behavior among seasons and breeds.

\section{Results}

The pigs were moved into the progeny test station after weaning and continued feeding for approximately 109 days, producing a total of 698671 original records from FIRE, and after application of the QC thresholds we had 597675 clean records for these animals. The respective minimum, maximum and average values of NV were 1, 41 and 8.48; of DFI were $0.011,6.513$ and $2.17 \mathrm{~kg}$; of OTPV were 1,29 and $7.21 \mathrm{~min}$; and of FIWPV were $0.011,1.063$ and $0.25 \mathrm{~kg}$.

\subsection{Growth performance}

The relationship between live body weight and age among the three breeds is shown in Fig. 1. A classic S-shaped growth curve tendency (slow-fast-slow) was observed in the three

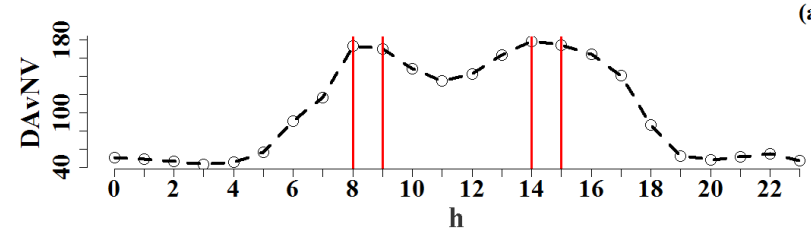

(a)

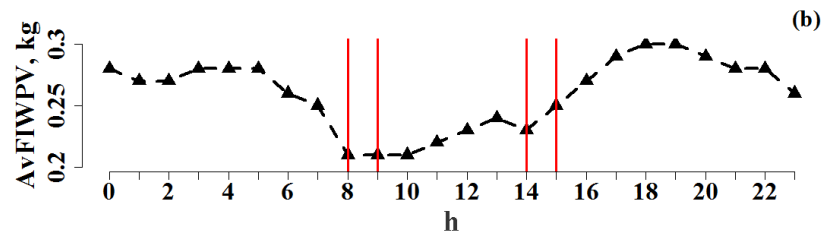

(c)

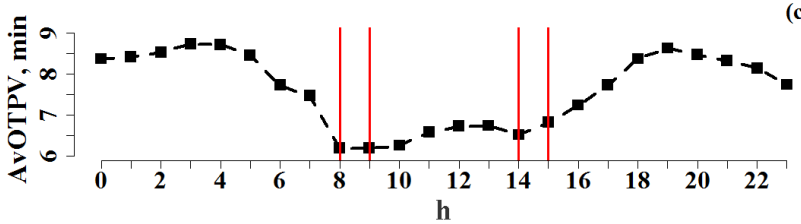

Figure 2. (a) Daily average number of visits (DAvNV) to FIRE; (b) average feed intake weight per visit (AvFIWPV), kg; and (c) average occupation time per visit (AvOTPV), min, over $24 \mathrm{~h}$ ( $x$ axes)

breeds and the growth curve was fitted using the logistic model. The logistic function for the three breeds is shown in Table 1 . The knee points of body weight and age (days) were defined as $K / 2$ and $\frac{\ln a}{b}$. The knee points of age (days) for Duroc, Landrace and Yorkshire were 152, 136 and 142 days, respectively, and the corresponding body weights were 69 , 62 and $64 \mathrm{~kg}$, respectively. The rapid growth periods for Duroc, Landrace and Yorkshire were found at the weights of $55 \mathrm{~kg}$ (137 days) to $103 \mathrm{~kg}$ (194 days), $57 \mathrm{~kg}$ (134 days) to $105 \mathrm{~kg}$ (192 days) and $59 \mathrm{~kg}$ (139 days) to $107 \mathrm{~kg}$ (194 days), respectively. Duroc had a smaller body weight when compared with the other two breeds during the first 120 days in the test station, but it catches up by the age of $\sim 220$ days, implying a slightly faster growth rate than the other two breeds. Due to fewer observations after the age of 240 days, a lot of volatility in the growth curve is shown. It is better to discard these data before further calculation and analysis.

\subsection{Effect of time of day on feed intake behavior during the whole test period}

Histogram distribution of the FIWPV is shown in Fig. A1. The minimum, mean and maximum values and variance of the FIWPV are $0.011,0.250,1.603$ and $0.047 \mathrm{~kg}$, respectively. A very large proportion of small intake weight per visit $(<200 \mathrm{~g})$ as well as a small proportion of large intake weight per visit are exhibited in the graph. This indicates that pigs prefer eating little and often then eating a lot of feed at fewer times in the day. 
Table 2. Interview of traits at each hour.

\begin{tabular}{|c|c|c|c|c|c|c|c|c|c|c|c|c|}
\hline Hour & 00:00 & 01:00 & 02:00 & 03:00 & 04:00 & 05:00 & 06:00 & 07:00 & 08:00 & 09:00 & $10: 00$ & 11:00 \\
\hline AvFIWPV, kg visit ${ }^{-1}$ & 0.28 & 0.27 & 0.27 & 0.28 & 0.28 & 0.28 & 0.26 & 0.25 & 0.21 & 0.21 & 0.21 & 0.22 \\
\hline AvOTPV, min visit ${ }^{-1}$ & 8.37 & 8.41 & 8.53 & 8.73 & 8.72 & 8.46 & 7.73 & 7.47 & 6.19 & 6.19 & 6.26 & 6.58 \\
\hline $\mathrm{AvNV}$ & 51.39 & 49.46 & 46.5 & 43.56 & 46 & 56.85 & 90.75 & 117.1 & 173.3 & 170.2 & 148.1 & 135.2 \\
\hline Hour & $12: 00$ & $13: 00$ & $14: 00$ & $15: 00$ & $16: 00$ & $17: 00$ & 18:00 & 19:00 & $20: 00$ & $21: 00$ & $22: 00$ & $23: 00$ \\
\hline AvFIWPV, kg visit ${ }^{-1}$ & 0.23 & 0.24 & 0.23 & 0.25 & 0.27 & 0.29 & 0.3 & 0.3 & 0.29 & 0.28 & 0.28 & 0.26 \\
\hline AvOTPV, min visit ${ }^{-1}$ & 6.73 & 6.74 & 6.52 & 6.82 & 7.24 & 7.73 & 8.37 & 8.63 & 8.47 & 8.32 & 8.14 & 7.74 \\
\hline AvNV & 142.4 & 163.6 & 178.5 & 174.3 & 164.5 & 141.4 & 87.13 & 52.93 & 48.62 & 51.89 & 54.93 & 47.85 \\
\hline
\end{tabular}

AvFIWPV: average feed intake weight per visit. AvOTPV: average occupation time per visit. AvNV: average number of visits to FIRE.

Proportional distribution of NV, OTPV and FIWPV over $24 \mathrm{~h}$ is presented in Fig. 2. The NV showed circadian patterns with two peaks of activity within $24 \mathrm{~h}$, in the morning and the afternoon. The top two peaks of daily average NV were from 08:00 (with an average of 173.3 visits $^{-1}$ ) to 09:00 (with an average of 170.2 visits) and in the afternoon from 14:00 (with an average of 178.5 visits) to 15:00 (with an average of 174.3 visits). However, the average FIWPV (0.21, $0.21,0.23,0.25 \mathrm{~kg})$ and the average OTPV $(6.19,6.19,6.52$, $6.82 \mathrm{~min})$, at these corresponding peaks (08:00, 09:00, 14:00 and 15:00, respectively) fall into some of the lowest weights or durations recorded in the 24-hour period (Fig. 2). To further investigate the possible impact factors of "breeds" and "pens", we showed the same distribution of the NV by breed in Fig. A2. The same pattern of two-peaked activity behavior at the same time was observed across the three breeds. More details about the NV, OTPV and FIWPV over the $24 \mathrm{~h}$ are shown in Table 2.

\subsection{Effective feed intake times for different pig breeds}

The relationship between feed intake weight and feed intake time for each breed is shown in Fig. 3. A linear relationship was observed increasing between intake weight and intake time at the initial stage (at 1-16 min), then accompanied by a slower feed speed (at 17-20 min) and, finally, feed intake ceased after $20 \mathrm{~min}$. The effective feed intake times for Duroc, Landrace and Yorkshire are about 19, 16 and $19 \mathrm{~min}$, respectively (Fig. 3) and the relevant feed intake speed was analyzed and shown in Fig. A3.

\subsection{Effect of seasons on feed intake behavior}

The daily feed intake weight for each breed that entered the test station at every season was plotted in Fig. 4. The statistical significance was defined as the $P$ value, less than 0.05 . The significant difference between autumn and winter to spring was detected by variance analysis and Tukey multiple comparison of means $(P$ value $=0.000009)$. Each breed displayed the same pattern by season. Pigs have a

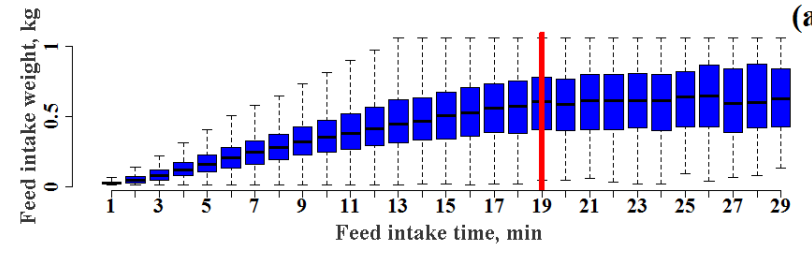

(a)

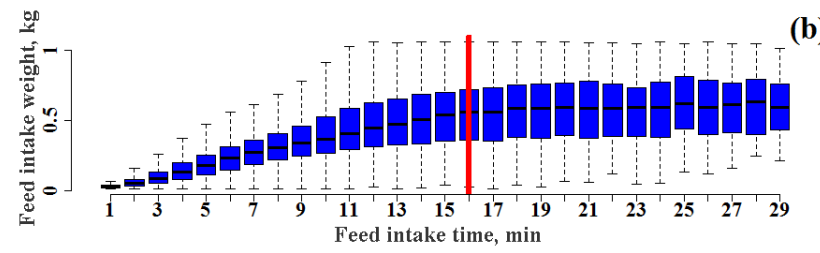

(b)

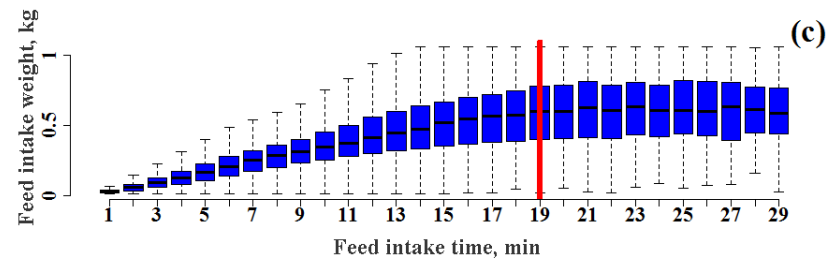

Figure 3. Effective feed intake times shown by red lines for (a) Duroc, $19 \mathrm{~min}$, (b) Landrace, $16 \mathrm{~min}$ and (c) Yorkshire, $19 \mathrm{~min}$.

lower daily feed intake weight in spring and winter than in autumn (Fig. 4).

\section{Discussion}

The main characteristic of American Duroc is that it has a fast growth rate (Smith et al., 1990). In our studies the average body weight of Duroc is lower than the other two breeds from entrance to 100 days in the test station. This implies that the average birth weight of Duroc is lower or its growth rate from birth to weaning is slower than the other two breeds. However, the live body weight of Duroc after 210 days catches up to the other two breeds, implying a slightly faster growth rate. We also observed that Duroc has a lower feed intake per day, probably because of its lighter body weight during the test periods. 
Nielsen et al. (1995) investigated feeding behaviors of 150 crossed male pigs using computerized feed-intake recording equipment and found a circadian rhythm in the weight of feed intake over $24 \mathrm{~h}$ and a feeding pattern with two peaks, in the morning and afternoon (Nielsen et al., 1995). Moreover, they also found that the higher the number of visits to FIRE per hour, the less the weight of feed intake per visit. Hoy et al. (2012) also reported the same phenomenon (Hoy et al., 2012). In our study, Yorkshire, Landrace and Duroc also showed a two-peaked activity pattern with the highest activity at 08:00-09:00 and 14:00-15:00 over $24 \mathrm{~h}$, accompanied by a low FIWPV at the highest peak of NV. Higher number of visits to FIRE with lower FIWPV at peak times means fiercer competition for access to the feeder in the housed population (Nielsen et al., 1995; Hyun et al., 1997). This suggests that a logical group size and equal access to the feeder are necessary.

Feed consumption is a major cost in pork production. Precision feed is aimed at optimizing feed intake. Rauw et al. (2006) reported that the average OTPV was about $19 \mathrm{~min}$ (e.g., Duroc, Rauw et al., 2006). In our study, effective feeding times for Duroc, Landrace and Yorkshire are about 19, 16 and 19 min, respectively. This result is the same as Rauw et al. (2006) previously reported for Duroc. After $20 \mathrm{~min}$, most pigs stay at the feeder and just play for fun or feed at a very low speed. The proportion of occupation time per visit that was longer than $20 \mathrm{~min}$ is about $3.2 \%$, decreasing the efficiency of FIRE. An improvement to FIRE would be to automatically expel pigs with occupation times longer than $20 \mathrm{~min}$. This is necessary to increase the capacity of FIRE. The average feeding time for Duroc and Yorkshire is longer than for Landrace, perhaps because the feed intake speed of these two breeds is lower than that of Landrace. Setting up the number of animals per pen more precisely in accordance to their breed would help to improve production efficiency. In addition, the welfare of animals is of considerable concern worldwide and continuous selection for increased efficiency may reduce the welfare of pigs (Hötzel et al., 2014). An optimization between production efficiency and the welfare of pigs is worth studying. Unfortunately, we could not realize this work due to the limitation of the established data set and more research still needs to be done.

Gourdine et al. (2006) reported that hot seasons can cause a reduction in the ADFI (average daily feed intake) compared with warm seasons (Gourdine et al., 2006). Heetkamp et al. (2005) also pointed out that high temperatures could cause a feed intake reduction (Heetkamp and Canh, 2005). Our data were recorded from 17 September 2014 to 27 May 2015 and therefore included autumn, winter and spring but not summer. We should also note that these pigs were kept in a controlled microclimate. The temperature was relatively stable, ranging from 16 to $20^{\circ} \mathrm{C}$, as described above. Theoretically, the impact of temperature on the ADFI should be eliminated because the present analysis indicates significant differences in ADFI between seasons $(P$ value $=0.000009)$,

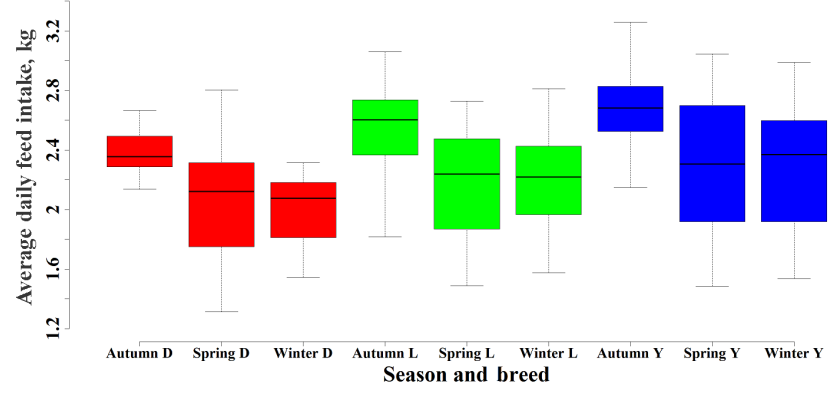

Figure 4. Comparison of daily feed intake weight at different seasons. The red, green and blue box plots represent Duroc (D), Landrace (L) and Yorkshire (Y), respectively. These breeds have a higher daily feed intake weight in autumn compared to spring and winter.

with a lower ADFI in spring and winter than in autumn, suggesting existence of a biological rhythm in pigs. We know autumn is a harvest season with plenty of food, while winter and spring are typically seasons with less food and lower temperatures. Animals may store energy in summer and autumn for overwintering. Wild pigs that are more active in summer and autumn show prominent peaks of farrowing in winter and spring, so wild pigs may store energy for farrowing in winter (Ultsch, 2008; Rosenfeld et al., 2009; Hartley et al., 2014). We hypothesize that wild pigs eat more in autumn to better handle the cold weather and less food in winter since ancient times, and these habits then slowly became an inner rhythm. The hypothesis that pigs show seasonal rhythm deserves further analysis.

\section{Conclusions}

In the present study, data were collected on three batches of pigs, a total of 823 individuals from three different breeds. We investigated their feeding behaviors and growth performance using several indexes including NV, OTPV, FIWPV and ADFI. A classic S-shaped growth curve was observed in the three breeds and the mean values for the NV, OTPV, FIWPV and ADFI were 8.48, $2.17 \mathrm{~kg}, 7.21 \mathrm{~min}$ and $0.25 \mathrm{~kg}$, respectively. We explored the relation between the NV, OTPV and FIWPV and found a two-peaked pattern of feed intake activity at certain times of day. We also discussed potential improvement in grouping animals into a pen, found that the most efficient feeding time is around $20 \mathrm{~min}$ and gave suggestions on the improvement of current feeders. Study of the ADFI showed a significant difference between seasons and consistent patterns in the three breeds. The results suggest that electronic feeding stations can not only help us improve production efficiency, but also offer us some information on feeding and social behaviors. With these results, we can formulate some parameters for precision feeding in order to modulate feeding and feed intake behavior of pigs and improve their production efficiency. 


\section{Appendix A}

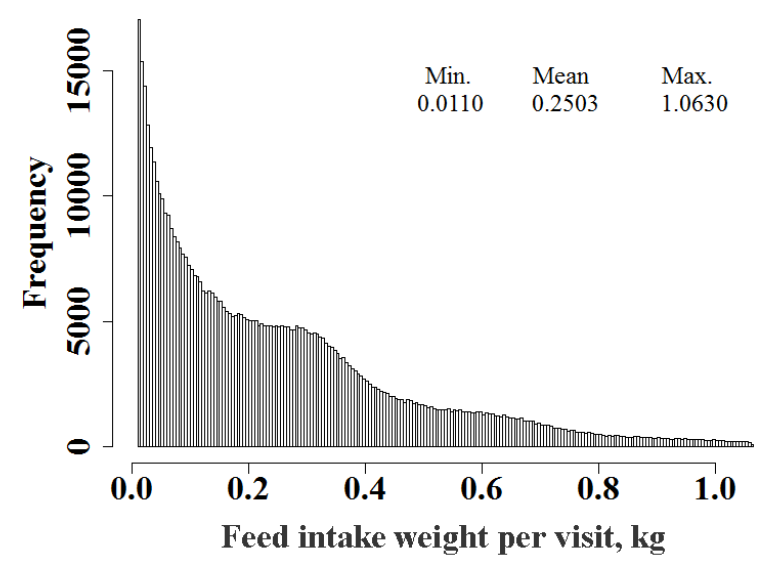

Figure A1. Histogram of feed intake weight per visit. Note: a very large proportion of results for small intake weight per visit, and a small proportion of results for large intake weight per visit. The $x$ axis represents feed intake weight per visit $(\mathrm{kg})$ and the $y$ axis shows the frequency of feed intake weight per visit (FIWPV).
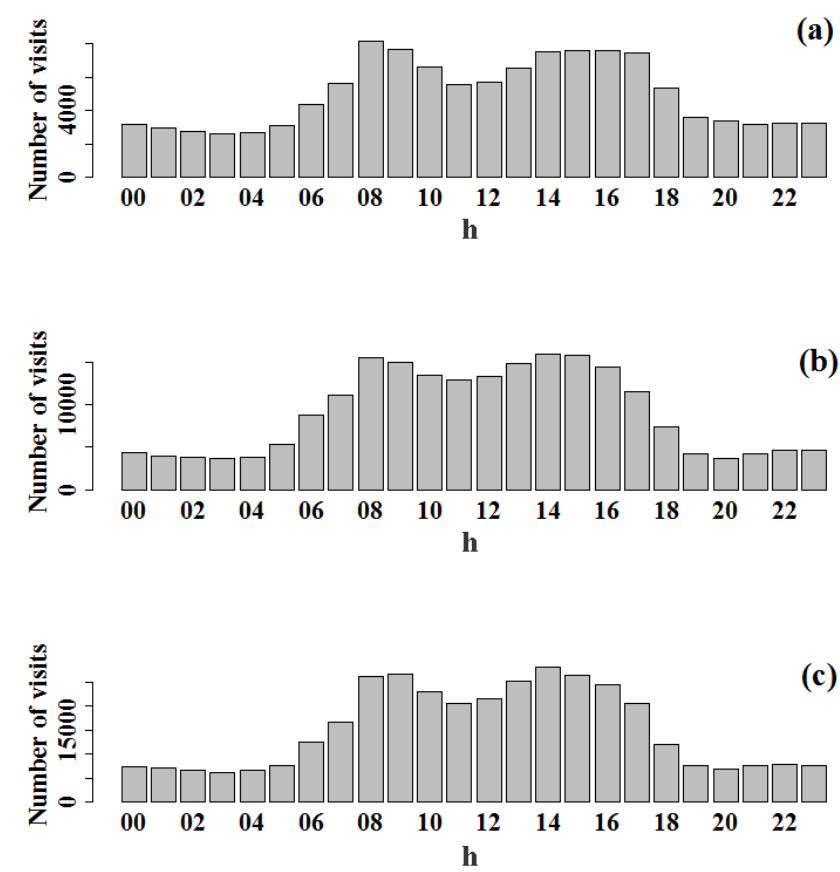

Figure A2. Distribution of the number of visits to FIRE over $24 \mathrm{~h}$. Note: the same pattern of two-peaked activity behaviors at the same time was observed across the three breeds. The $x$ axis represents hour, the $y$ axis represents the number of visits to FIRE for the three batches of pigs during the whole test period and (a), (b) and (c) represent Duroc, Landrace and Yorkshire, respectively.

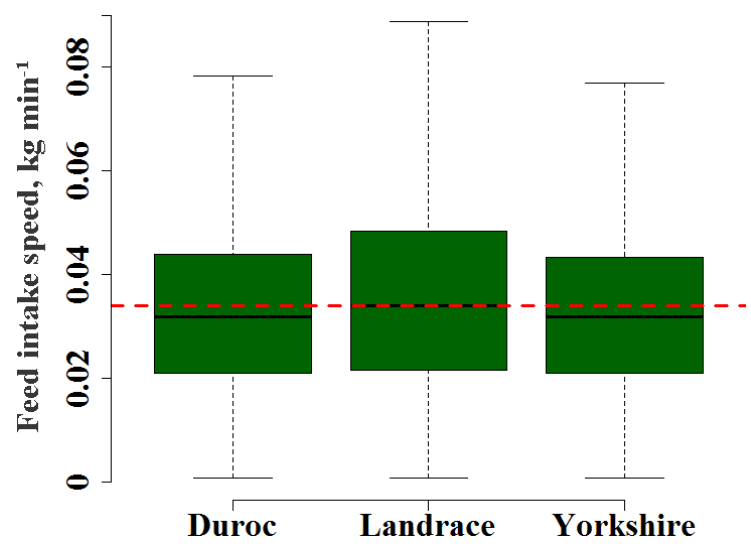

Figure A3. Feed intake speed for the three breeds. The $x$ axis represents the breed and the $y$ axis represents the feed intake speed. The red dotted line shows the median feed intake speed value of Landrace. 
Competing interests. The authors declare that they have no conflict of interest.

Author contributions. Zhiyan Zhang and Lusheng Huang conceived this study. Wenshui Xin performed the analysis and wrote the paper with Xinjian Li. Nengshui Ding, Feng Zhang and Guorong Yan contributed to sample collection and a part of the data analysis.

Acknowledgements. This study was supported by the Project of Science and Technology in Jiangxi Province (20143ACF60003), the Pig Industry Technology System Innovation Team Project in Henan Province (S2012-06) and the Innovation Fund for Graduate Students in Jiangxi Province (YC2015-B027). The authors thank the Henan Xinda Livestock Company Ltd., China, for providing the data.

Edited by: M. Mielenz

Reviewed by: M. Fang and one anonymous referee

\section{References}

Abijaoudé, J., Morand-Fehr, P., Tessier, J., Schmidely, P., and Sauvant, D.: Diet effect on the daily feeding behaviour, frequency and characteristics of meals in dairy goats, Livest. Prod. Sci., 64, 29-37, 2000.

Basarab, J., Price, M., Aalhus, J., Okine, E., Snelling, W., and Lyle, $\mathrm{K}$.: Residual feed intake and body composition in young growing cattle, Can. J. Anim. Sci., 83, 189-204, 2003.

Chapinal, N., Veira, D., Weary, D., and Von Keyserlingk, M.: Technical note: Validation of a system for monitoring individual feeding and drinking behavior and intake in group-housed cattle, J. Dairy Sci., 90, 5732-5736, 2007.

Chapinal, N., Ruiz-de-la-Torre, J.L., Cerisuelo, A., Baucells, M. D., Gasa, J., and Manteca, X.: Feeder use patterns in group-housed pregnant sows fed with an unprotected electronic sow feeder (Fitmix), J. Appl. Anim. Welfare Sci., 11, 319-336, 2008.

Eigenberg, R., Hahn, G., Nienaber, J., Brown-Brandl, T., and Spiers, D.: Development of a new respiration rate monitor for cattle, $\mathrm{T}$. ASAE, 43, 723-728, 2000.

Fuller, M., Franklin, M., McWilliam, R., and Pennie, K.: The responses of growing pigs, of different sex and genotype, to dietary energy and protein, Anim. Sci., 60, 291-298, 1995.

Gipson, T., Goetsch, A., Detweiler, G., and Sahlu, T.: Effects of feeding method, diet nutritive value and physical form and genotype on feed intake, feeding behavior and growth performance by meat goats, Small Ruminant Res., 71, 170-178, 2007.

Goetsch, A., Gipson, T., Askar, A., and Puchala, R.: Invited review: Feeding behavior of goats, J. Anim. Sci., 88, 361-373, 2010.

Gourdine, J., Bidanel, J., Noblet, J., and Renaudeau, D.: Effects of season and breed on the feeding behavior of multiparous lactating sows in a tropical humid climate, J. Anim. Sci., 84, 469-480, 2006.

Griffin, D.: How health status and environment influence the feeding behavior of feedlot cattle, Intermountain Nutrition Conference, Utah State University, 117-124, 2001.
Hartley, S. B., Goatcher, B. L., and Sapkota, S. K.: Movements of Wild Pigs in Louisiana and Mississippi, Usgs Professional Paper, 2014.

Heetkamp, M. and Canh, T. T.: Thermal behaviour of growing pigs in response to high temperature and humidity, Appl. Anim. Behav. Sci., 91, 1-16, 2005.

Hötzel, M. J., Appleby, M. C., Weary, D. M., and Sandøe, P.: Improving Farm Animal Welfare: Is Evolution or Revolution Needed in Production Systems?, Dilemmas in Animal Welfare, 2014, 67-83, 2014.

Hoy, S., Schamun, S., and Weirich, C.: Investigations on feed intake and social behaviour of fattening pigs fed at an electronic feeding station, Appl. Anim. Behav. Sci., 139, 58-64, 2012.

Hyun, Y., Ellis, M., McKeith, F., and Wilson, E.: Feed intake pattern of group-housed growing-finishing pigs monitored using a computerized feed intake recording system, J. Anim. Sci. 75, 14431451, 1997.

Kelly, A., McGee, M., Crews, D., Sweeney, T., Boland, T., and Kenny, D.: Repeatability of feed efficiency, carcass ultrasound, feeding behavior, and blood metabolic variables in finishing heifers divergently selected for residual feed intake, J. Anim. Sci., 88, 3214-3225, 2010.

Korthals, R.: Evaluation of space requirements for swine finishing feeders, T. ASAE, 43, 395-398, 2000.

Nielsen, B. L., Lawrence, A. B., and Whittemore, C. T.: Effect of group size on feeding behaviour, social behaviour, and performance of growing pigs using single-space feeders, Livest. Prod. Sci., 44, 73-85, 1995.

Puma, M. C., Xin, H., Gates, R. S., and Burnham, D. J.: An instrumentation system for studying feeding and drinking behavior of individual poultry, Appl. Eng. Agric., 17, 365-374, 2001.

Rauw, W., Soler, J., Tibau, J., Reixach, J., and Gomez Raya, L.: Feeding time and feeding rate and its relationship with feed intake, feed efficiency, growth rate, and rate of fat deposition in growing Duroc barrows, J. Anim. Sci., 84, 3404-3409, 2006.

Rosenfeld, A., Hinkle, C. R., and Epstein, M. B.: Influence of Habitat Attributes on Removal of Feral Hogs from Merritt Island National Wildlife Refuge and Kennedy Space Center, Florida, in: Wild Pigs Biology, Damage, Control Techniques And Management, Savannah River National Laboratory Aiken, South Carolina, 387-400, 2009.

Smith, W., Pearson, G., and Purchas, R.: A comparison of the Duroc, Hampshire, Landrace, and Large White as terminal sire breeds of crossbred pigs slaughtered at $85 \mathrm{~kg}$ liveweight: 1 . Performance and carcass characteristics, New Zeal. J. Agr. Res., 33, 89-96, 1990.

Sołtysiak, T. and Nogalski, Z.: The effects of social hierarchy in a dairy cattle herd on milk yield, Pol. J. Natur. Sc., 25, 22-30, 2010.

Ultsch, G. R.: Ecology and physiology of hibernation and overwintering among freshwater fishes, turtles, and snakes, Biol. Rev., 64, 435-515, 2008.

Val-Laillet, D., de Passillé, A. M., Rushen, J., and von Keyserlingk, M. A.: The concept of social dominance and the social distribution of feeding-related displacements between cows, Appl. Anim. Behav. Sci., 111, 158-172, 2008.

Walker, S., Smith, R., Routly, J., Jones, D., Morris, M., and Dobson, H.: Lameness, activity time-budgets, and estrus expression in dairy cattle, J. Dairy Sci., 91, 4552-4559, 2008. 
Xihong, G., Naijun, S., Fei, Z., Youyu, S., Cuiling, Z., and Dengyun, L.: Preliminary study on the establishment of Logistic model by Excel, China Plant Protection, 31, 13-15, 2011.

Yongqiang, Q., Zhenyuan, S., Lei, H., and Guansheng, J.: Simulation of Clonal Growth Curve Based on Nonlinear Models of Logistic and Gompertz for Buffalograss (Buchloe dactyloides (Nutt. Engelm Texoka), Sci. Agricult. Sinica, 44, 2252-2259, 2011. 